\title{
Creation of entangled states of distant atoms by interference
}

\author{
C. Cabrillo, ${ }^{1}$ J. I. Cirac, ${ }^{2}$ P. García-Fernández, ${ }^{1}$ and P. Zoller ${ }^{2}$ \\ ${ }^{1}$ Instituto de Estructura de la Materia, Serrano 123, E-28006 Madrid, Spain \\ ${ }^{2}$ Institut für Theoretische Physik, Universität Innsbruck, A-6020 Innsbruck, Austria
}

(Received 28 September 1998)

\begin{abstract}
We propose a scheme to create distant entangled atomic states. It is based on driving two (or more) atoms with a weak laser pulse, so that the probability that two atoms are excited is negligible. If the subsequent spontaneous emission is detected, the entangled state is created. We have developed a model to analyze the fidelity of the resulting state as a function of the dimensions and location of the detector, and the motional properties of the atoms. [S1050-2947(99)04502-3]
\end{abstract}

PACS number(s): 03.65.Bz, 42.50.Vk

\section{INTRODUCTION}

The preparation of entangled atomic states is one of the goals of atomic physics and quantum optics. These states are a key ingredient for studying some fundamental issues of quantum mechanics [1], as well as for certain applications related to quantum information [2]. Methods proposed so far to "engineer" entanglement between atoms in the laboratory are based on achieving and controlling an effective interaction between the atoms that are to be entangled. Typically, these interactions are mediated by the electromagnetic field. For example, in cavity QED, two atoms can be entangled if they both interact with the same cavity mode [3]. This coupling of the two atoms to the field mode can be simultaneous or sequential (that is, one atom interacts first with the cavity mode, and then the other one). With trapped ions, entangled states can be produced by using the Coulomb repulsion between the ions, together with some laser couplings [4]. With these methods, it is always necessary that the atoms interchange some particles (photons) or that they are very close to each other.

In this paper we propose a scheme to prepare entangled atomic states using a different approach. In particular, the entangled state is not produced by an effective interaction between the atoms, but rather by an interference effect and state projection accompanying a measurement. Imagine that we have two atoms $A$ and $B$, situated in distant locations, both in an excited state $|0\rangle$. These atoms may decay to the state $|1\rangle$ due to spontaneous emission, producing one photon. A detector is placed at half the way between the atoms. After some time, if the detector clicks and we cannot distinguish from where the detected photon came, we will have produced an entangled state

$$
|\Psi\rangle=\frac{1}{\sqrt{2}}\left(|0\rangle_{A}|1\rangle_{B}+e^{i \phi}|1\rangle_{A}|0\rangle_{B}\right)
$$

where $\phi$ is a fixed phase. Entanglement is then achieved as a consequence of two facts: first, the impossibility to determine from the detection event which atom emitted the photon, second, the projection postulate in quantum mechanics, which indicates that after the detection the state of the atoms is projected onto the one which is compatible with the outcome of the measurement. The first effect is precisely the one that would give rise to interference fringes at the detector position if one would repeat several times the experiment, as it has been shown by the NIST group at Boulder $[5,6]$. The second effect has been used, for example, in the preparation of nonclassical states of a cavity mode [7]. Using this method to prepare entangled states, the atoms do not need to interact, and no interchange of particles (photons) is required. In fact, the entanglement can be produced (in principle) in a time which is half the distance between the atoms divided by the speed of light.

In practice, the method described above might not be very useful. First, it is very unlikely that the photon emitted by one of the atoms is detected. Second, and more important, even if one photon is detected, the second atom will eventually decay to the ground state thus yielding the state $|1\rangle_{A}|1\rangle_{B}$, which is not entangled. Here we will analyze in some detail how an experiment can be performed in a realistic setup. The idea is to use two three-level atoms with a Lambda configuration (see Fig. 1). The states $|0\rangle$ and $|1\rangle$ are the two ground states, so that once the state (1) is prepared, it will stay. Both atoms are initially prepared in the state $|0\rangle$. The excitation is achieved by using a very short laser pulse, which (with a small probability) excites one of the two atoms to level $|2\rangle$. If following the excitation a spontaneously emitted photon is detected, an entangled state of the two atoms

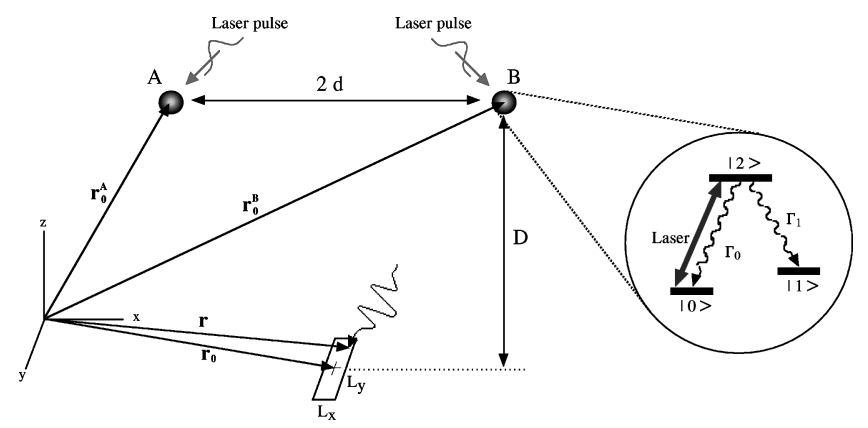

FIG. 1. Sketch of the experimental setup as well as of the internal level structure of the atoms corresponding to the proposed experiment. 
will be produced. The method presented here seems particularly timely, in view of the spectacular experimental progress reported by the NIST group of observation of interference fringes of the light emitted by two independent atoms [5]. In fact, the same experimental setup could be used to prepare atomic entangled states using our proposal.

In order to estimate the conditions that must be fulfilled to create an entangled state, we have developed a theoretical model describing the whole process of laser excitation of the two atoms, spontaneous emission of a photon, and detection. The idea is to represent the detector as a collection of atoms, and then to use master equation methods to describe the projection occurring when a detection event is recorded. In this way, the electromagnetic field does not appear explicitly in the formulas, making the calculations simpler. We emphasize that the model is equivalent to the one in which the whole state of the electromagnetic field is taken into account at all times, and the measurement projects its state along with the state of the atoms. This model can be easily generalized to other situations in which there are more atoms present, yielding entangled states of more than two atoms.

The paper is organized as follows. In Sec. II we explain qualitatively the details of our proposal and discuss the main results, and some of the practical problems. In Sec. III we present the theoretical model. In Sec. IV we obtain an analytical formula for the fidelity of the final state as a function of the physical parameters involved in the problem. Finally, in Sec. IV we discuss the results and point out some possible generalizations.

\section{QUALITATIVE DESCRIPTION}

Let us consider two atoms $A$ and $B$ separated by a distance $2 d$. Each of the atoms has an internal structure which can be described in terms of a three-level Lambda system (see Fig. 1). It consists of two ground levels $|0\rangle$ and $|1\rangle$, and an excited state $|2\rangle$. A photodetector is located at a distance $D$ from the segment connecting atoms $A$ and $B$ (see Fig. 1). The detector is sensitive to photons of wavelength $\lambda_{1}$ (and/or polarization) corresponding to the transition $|2\rangle \rightarrow|1\rangle$, which is characterized by a spontaneous emission rate $\Gamma_{1}$. It is not, however, sensitive to the ones corresponding to the other transition.

Both atoms are initially prepared in the state $|0\rangle$. Then, they are driven by a very short laser pulse on resonance with the transition $|2\rangle \leftrightarrow|0\rangle$. As a consequence, sometimes one of the atoms (or both) will spontaneously emit a photon of wavelength $\lambda_{1}$, which might be recorded at the photodetector. Most of the times, no photon will be detected after a waiting time $t \gg \Gamma_{1}$. In such a case, the atoms are pumped back to the original state $|0\rangle$, and the experiment is repeated until the detector clicks. Once this occurs, the state of both atoms will be described by a density operator $\rho_{A, B}$. The goal is to obtain a state as close as possible to the maximally entangled state (1) where $\phi$ is a phase that does not change from experiment to experiment. That is, we wish to obtain a fidelity

$$
F=\left\langle\Psi\left|\rho_{A, B}\right| \Psi\right\rangle,
$$

close to 1 .
The physical idea is that the laser pulse prepares a superposition state of the two atoms, which apart from the state $|0\rangle_{A}|0\rangle_{B}$ also contains a coherent superposition of the states $|0\rangle_{A}|2\rangle_{B}$ and $|2\rangle_{A}|0\rangle_{B}$. Detection of a photon implies that a transition $|2\rangle \rightarrow|1\rangle$ has taken place in one of the atoms, producing a photon of wavelength $\lambda_{1}$ that is detected. The term $|0\rangle_{A}|0\rangle_{B}$ will thus be projected out from the atomic state, since it is incompatible with that event (the state $|1\rangle$ of one of the atoms must be present in the atomic state). Moreover, given the fact that the detector cannot distinguish among photons emitted by different atoms, the superposition of the states $|0\rangle_{A}|2\rangle_{B}$ and $|2\rangle_{A}|0\rangle_{B}$ will be transformed into a superposition of the states $|0\rangle_{A}|1\rangle_{B}$ and $|1\rangle_{A}|0\rangle_{B}$, i.e., it will be close to the entangled state (1).

In order to obtain an entangled state close to the ideal Bell state (1), several conditions have to be satisfied. (i) First, the laser pulse has to be such that the probability of exciting both atoms to the state $|2\rangle_{A}|2\rangle_{B}$ has to be much smaller than the probability of exciting the relevant coherent superposition. Otherwise, it may happen that although we detect a photon emitted by one of the atoms, the other atom also emits a photon albeit in another direction which is not detected; this would spoil the fidelity $F$ since the final state of this process would be $|1\rangle_{A}|1\rangle_{B}$. In order to avoid this problem one must use a sufficiently weak or short laser pulse. In that case, the probability of exciting two atoms $\epsilon^{2}$ is of the order of the square of the probability of exciting only one atom $\simeq 2 \epsilon$. By choosing $\epsilon \ll 1$ one avoids the two-atom excitation. Notice, however, that the laser beam cannot be too weak since it would take a very long time to detect one spontaneously emitted photon, given that the detection probability is proportional to $\epsilon$. (ii) Second, the detector has to be sufficiently small. At each point of the detector the phase $\phi$ will have a different value spoiling the fidelity since a detection does not specify the exact location of the event, and therefore the exact phase is unknown. Thus, the detector has to be such that at all points the phase is practically the same. In order to estimate the required size of the detector surface one can use the analogy between the situation considered here and the double slit experiment: the distance traveled by a photon coming from one atom or the other will be somewhat different at different positions, and therefore the accumulated phase depends on the position in which it is detected. The phase will be essentially constant over regions where the corresponding interference fringes have a constant visibility. Thus, the length $L_{x}$ of the detector along the $X Z$ plane has to be much smaller than the interfringe distance $L_{x} \ll \lambda_{1} D / d$. However, the detection probability is proportional to the size of the detector and therefore we cannot take $L_{x}$ arbitrarily small. (iii) Furthermore, the dynamics of the atoms during the absorption emission cycle will also affect the final fidelity. In fact, every absorption or emission of photons by an atom is always accompanied by a recoil, which changes the atomic motional state. This leaves a trace of which atom has emitted the photon, thus also destroying the entanglement. In order to avoid this problem, one has to find a way "not to leave information about the motional states behind." This can be done, for example, by using trapped particles and operating in the Lamb-Dicke limit, where the recoil energy does not suffice to change the atomic motional state (similar to the Mösbauer effect). However, the extent to which this 
effect can be reduced will also depend on the temperature of the atoms in the trap, as well as on the propagation directions of the laser beams.

In the following sections we will solve in detail a theoretical model to answer all of these questions. Our result is a simple formula for the fidelity in which these effects are clearly separated. We consider a situation where the atoms are trapped in identical isotropic harmonic potentials, characterized by a frequency $\nu$ and initial temperature $T$. We obtain

$$
F=\frac{\cos ^{2}\left(\theta_{\text {las }}\right)}{2}\left(1+F_{\text {geo }} F_{\text {dyn }}\right),
$$

where $\theta_{\text {las }}$ is the pulse area (Rabi frequency times time), and $F_{\text {dyn }}$ and $F_{\text {geo }}$ represent a dynamical and a geometrical factor, respectively. More specifically,

$$
F_{\mathrm{geo}}=\operatorname{sinc}\left[\frac{d L_{x}}{2 \lambda_{1} \sqrt{d^{2}+D^{2}}}\right]
$$

where $\operatorname{sinc}(x)=\sin (x) / x$. We also have

$$
\begin{aligned}
F_{\mathrm{dyn}}= & \int_{0}^{\infty} d \tau e^{-\tau} \exp \left\{-2 \eta^{2} \operatorname{coth}\left(\frac{\hbar \nu}{2 k_{B} T}\right)\right. \\
& \left.\times\left[1-\cos (\chi) \cos \left(\frac{\nu \tau}{\Gamma}\right)\right]\right\} .
\end{aligned}
$$

Here, $\eta=2 \pi a_{\mathrm{tp}} / \lambda_{1}$ is the so-called Lamb-Dicke parameter, with $a_{\mathrm{tp}}=\sqrt{\hbar / 2 m \nu}$ the size of the harmonic trapping potential ground state, $\Gamma$ is the total spontaneous emission rate from level $|2\rangle$, and $\chi$ is the angle between the propagation direction of the laser acting on an atom and the line that connects the atom with the center of the detector (we take this angle to be the same for atoms $A$ and $B$ ).

The first factor in Eq. (3) accounts for the effects due to the laser excitation. That is, when $\theta_{\text {las }}$ increases, the fidelity decreases due to the fact that both atoms may be simultaneously excited. The geometrical factor is related to the size of the detector with respect to the interfringe distance. For small detectors compared with such a distance, this factor approaches 1. Finally, the dynamical factor shows that the fidelity increases for small Lamb-Dicke parameters and low temperatures, and depends on the ratio $\nu / \Gamma$ as well as the direction of the lasers. The highest fidelity occurs for $\cos (\chi) \simeq 1$ and $\eta^{2} \operatorname{coth}\left(\hbar \nu / 2 k_{B} T\right) \ll(\Gamma / \nu)^{2}$. The first condition means that the laser direction and the direction of the photon emitted and recorded at the detector has to be practically the same. In that case the recoil given by the laser is compensated by the recoil experienced by the atom in the spontaneous emission process that is monitored at the photodetector, and therefore no trace of which atom has emitted is left behind. Under such circumstances a $\nu \ll \Gamma$ (weak confinement) is needed so that the atom does not have time to oscillate in the trap before the spontaneous emission takes place-this would destroy the compensation of the recoils between the absorption-emission process. In these limits we can approximate

$$
\begin{gathered}
F_{\mathrm{geo}} \simeq 1-\frac{1}{6}\left[\frac{d L_{x}}{2 \lambda_{1} \sqrt{d^{2}+D^{2}}}\right]^{2}, \\
F_{\mathrm{dyn}} \simeq 1-2 \eta^{2} \operatorname{coth}\left(\frac{\hbar \nu}{2 k_{B} T}\right)\left(\frac{\nu}{\Gamma}\right)^{2} .
\end{gathered}
$$

On the other hand, under conditions of strong confinement $(\Gamma \ll \nu)$ although it is not possible to compensate for the harmful effect of the recoil by choosing the laser propagation direction, the dynamical factor can be very close to one in the Lamb-Dicke limit $(\eta \ll 1)$. In particular, for $\eta^{2} \operatorname{coth}\left(\hbar \nu / 2 k_{B} T\right) \ll 1$ we have

$$
F_{\mathrm{dyn}} \simeq 1-2 \eta^{2} \operatorname{coth}\left(\frac{\hbar \nu}{2 k_{B} T}\right)
$$

\section{MODEL}

\section{A. Master equation for the atoms and photodetector}

We consider two identical atoms $A$ and $B$, centered at positions $\mathbf{r}_{0}^{A}$ and $\mathbf{r}_{0}^{B}$, separated by a distance $2 d=\left|\mathbf{r}_{0}^{A}-\mathbf{r}_{0}^{B}\right|$. Each of the atoms has an internal structure which can be described in terms of a three-level Lambda system (see Fig. 1 ). It consists of two ground levels $|0\rangle$ and $|1\rangle$, and an excited state $|2\rangle$. Spontaneous emission from level $|2\rangle$ to both ground levels is possible, and is characterized by the rates $\Gamma_{0,1}$ and wave vectors $\mathbf{k}_{0,1}(\Omega)$, where $\Omega$ represents a direction and $\Gamma=\Gamma_{0}+\Gamma_{1}$ the total decay width of the excited state.

A detector of surface dimensions $S=L_{x} L_{y}$ and efficiency $\eta_{D}$ is situated in the $X Y$ plane, at a distance $D$ from the segment connecting atoms $A$ and $B$. The center of the detector $\mathbf{r}_{0}$ and the center of atoms $A$ and $B$ define the $X Z$ plane. We will describe the detector as a collection of independent point atoms located at position $\mathbf{r}$, with $\mathbf{r}$ varying along the detector surface [8]. These atoms have two internal discrete levels $|g\rangle$ and $|e\rangle$, which are resonant with the wavelength $\lambda_{1}=2 \pi / k_{1}$. The level $|e\rangle$ is monitored for population at time intervals $\delta t$ which we will take to be sufficiently small so that the atomic dynamics can be neglected during that time. The level $|e\rangle$ has a width $\gamma$ : for sufficiently large values of $\gamma$ our model corresponds to a broadband detector, whereas for small values it corresponds to a narrowband detector. The results will be independent of the specific value of $\gamma$. We will concentrate on a given atom $C$ of the detector coupled to the quantized electromagnetic field, which in turn is coupled to atoms $A$ and $B$. We will calculate the state in which those atoms are left when the atom $C$ is found in the state $|e\rangle$, and we will add incoherently the contributions corresponding to different detection times and different positions r. In such a way we will be finally able to derive an expression for the density operator of atoms $A$ and $B$ conditioned to the observation of a click of the detector. 
Using standard methods of quantum optics, one can trace out the electromagnetic field and obtain a master equation for the atoms $A, B$, and $C$

$$
\frac{d}{d t} \rho=\left[\mathcal{L}^{C}+\sum_{\alpha=A, B}\left(\mathcal{L}^{\alpha}+\mathcal{S}^{\alpha, C}+\mathcal{J}^{\alpha, C}\right)\right] \rho,
$$

where $\mathcal{L}^{\alpha}$ denotes the Liouvillian superoperator describing the evolution of atom $\alpha$ alone, and

$$
\begin{gathered}
\mathcal{S}^{\alpha, C} \rho=-i \frac{\tilde{\gamma}}{2} G\left(\left|\mathbf{r}^{\alpha}-\mathbf{r}\right|\right)\left(\sigma_{e g}^{C} \otimes \sigma_{12}^{\alpha}+\sigma_{g e}^{C} \otimes \sigma_{21}^{\alpha}\right) \rho+\text { H. c., } \\
\mathcal{J}^{\alpha, C} \rho=\tilde{\gamma} \int \frac{d \Omega}{4 \pi} e^{-i \mathbf{k}(\Omega) \cdot \mathbf{r}^{\alpha}} \sigma_{12}^{\alpha} \rho \sigma_{e g}^{C} e^{i \mathbf{k}(\Omega) \cdot \mathbf{r}}+\text { H. c. }
\end{gathered}
$$

with $\sigma_{i j}^{\alpha}=|i\rangle_{\alpha}\langle j|$ (superscripts indicate the atom, whereas subscripts indicate the states). Here and in the following we will use the symbol $\otimes$ (tensor product) whenever we feel that it clarifies the corresponding expression. The vectors $\mathbf{r}^{A}$ and $\mathbf{r}^{B}$ are the position operators of the atoms $A$ and $B$, while the vector $\mathbf{r}$ is treated as a $c$ number. The presence of the factor $G(\mathbf{r})=-\exp \left(i k_{1}|\mathbf{r}|\right) /\left(k_{1}|\mathbf{r}|\right)$ is due to the dipole-dipole interaction (real part) and reabsorption (imaginary part) between atoms $A, B$, and $C, \tilde{\gamma}$ giving the typical strength of this interaction. These two terms give rise to the excitation of atom $C$ via a photon absorption from atoms $A$ and/or $B$, which leads to a detection event. We have assumed $k\left(d^{2}\right.$ $\left.+D^{2}\right)^{1 / 2} \gg 1$, so that only the far-field part contributes to the dipole-dipole interaction.

The Liouvillian action on atom $C$ (detector) is given by

$$
\mathcal{L}^{C} \rho=-\frac{\gamma}{2}\left(\sigma_{e e}^{C} \rho+\rho \sigma_{e e}^{C}\right)+\gamma \sigma_{g e}^{C} \rho \sigma_{e g}^{C} .
$$

In the absence of laser excitation, we have $(\alpha=A, B)$

$$
\begin{aligned}
\mathcal{L}^{\alpha}= & \frac{1}{i \hbar}\left[H_{\mathrm{tp}}^{\alpha}, \rho\right]-\frac{\Gamma}{2}\left(\sigma_{22}^{\alpha} \rho+\rho \sigma_{22}^{\alpha}\right) \\
& +\Gamma_{0} \int \frac{d \Omega}{4 \pi} N_{0}(\Omega) e^{-i \mathbf{k}_{0}(\Omega) \cdot \mathbf{r}^{\alpha}} \sigma_{02}^{\alpha} \rho \sigma_{20}^{\alpha} e^{i \mathbf{k}_{0}(\Omega) \cdot \mathbf{r}^{\alpha}} \\
& +\Gamma_{1} \int \frac{d \Omega}{4 \pi} N_{1}(\Omega) e^{-i \mathbf{k}_{1}(\Omega) \cdot \mathbf{r}^{\alpha}} \sigma_{12}^{\alpha} \rho \sigma_{21}^{\alpha} e^{i \mathbf{k}_{1}(\Omega) \cdot \mathbf{r}^{\alpha}} .
\end{aligned}
$$

Here, $H_{\mathrm{tp}}$ is the Hamiltonian describing the motion of an atom in an isotropic harmonic potential of frequency $\nu$, and $N_{0}$ and $N_{1}$ describe the dipole emission pattern corresponding to transitions $|2\rangle \rightarrow|0\rangle$ and $|2\rangle \rightarrow|1\rangle$, respectively.

The master equation (8) can be solved formally as

$$
\begin{aligned}
\rho(t)= & e^{\left(\mathcal{L}^{A}+\mathcal{L}^{B}+\mathcal{L}^{C}\right)\left(t-t_{0}\right)} \rho\left(t_{0}\right)+\int_{t_{0}}^{t} d \tau e^{\left(\mathcal{L}^{A}+\mathcal{L}^{B}+\mathcal{L}^{C}\right)(t-\tau)} \\
& \times\left[\mathcal{S}^{A, C}+\mathcal{J}^{A, C}+\mathcal{S}^{B, C}+\mathcal{J}^{B, C}\right] \rho(\tau)
\end{aligned}
$$

This integral equation can be iterated to obtain a formal expansion in terms of $\mathcal{S}$ and $\mathcal{J}$. Since each of these terms scales as $1 / k\left(d^{2}+D^{2}\right)^{1 / 2} \ll 1$, we can stop at the first nonvanishing order of the equation. The even terms of the expansion correspond to physical processes in which excitations (photons) are interchanged between atoms $A$ and $C$ (or $B$ and $C$ ). We have not included in Eq. (8) the (dipole-dipole) interactions between atoms $A$ and $B$ which would give rise to processes describing photon exchange, because they correspond to a very small correction of the order of $1 / k d \ll 1$ to the final result. Note that we should only consider the case in which atom $C$ is detected in $|e\rangle$, which can only occur if a photon coming from $A$ or $B$ is absorbed; that is, the first nonvanishing process in our expansion will correspond to the emission of a photon from atom $A$ or $B$ subsequently absorbed by atom $C$. This will give a contribution of the order $1 / k^{2}\left(d^{2}\right.$ $\left.+D^{2}\right)$. Processes in which more than one photon are interchanged between atoms $A$ (or $B$ ) and $C$, or in which (apart from the photon absorbed by $C$ ) other photons are interchanged between atoms $A$ and $B$ would give higher order contributions, at least of the order of $1 / k^{4}\left(d^{2}+D^{2}\right)^{2}$ or $1 / k^{4}\left(d^{2}+D^{2}\right) d^{2}$, respectively.

\section{B. Initial state of atoms $A$ and $B$ : Laser interaction}

So far, we have ignored the initial state of atoms $A$ and $B$. Let us assume that they are driven by a very short laser pulse of duration $t_{\text {las }} \ll \Gamma^{-1}, \nu^{-1}$. The state of atom $\alpha$ after the interaction is

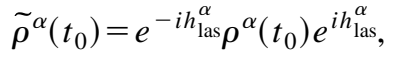

where $\rho^{\alpha}\left(t_{0}\right)=\sigma_{00}^{\alpha} \otimes \rho_{\text {tp }}^{\alpha}\left(t_{0}\right)$, with

$$
\rho_{\mathrm{tp}}^{\alpha}\left(t_{0}\right) \propto \exp \left(-H_{\mathrm{tp}}^{\alpha} / k_{B} T\right)
$$

being the initial motional state corresponding to a thermal distribution at temperature $T$ in the trapping potential, and $e^{-i h_{\text {las }}^{\alpha}}$ acts in the subspace $\operatorname{span}\left\{|0\rangle_{\alpha},|2\rangle_{\alpha}\right\}$ as

$$
e^{-i h_{\text {las }}^{\alpha}}=\cos \left(\theta_{\text {las }}\right)-i \sin \left(\theta_{\text {las }}\right)\left[\sigma_{20}^{\alpha} e^{i \mathbf{k}^{\alpha} \cdot \mathbf{r}^{\alpha}}+\text { H. c. }\right]
$$

Here, $\theta_{\text {las }}$ is the rotation angle due to the laser interaction and $\mathbf{k}^{\alpha}$ the laser wave vector acting on atom $\alpha$.

According to these equations, the effect of the laser on each of the atoms is twofold: on one hand, it excites a superposition of the internal states $|0\rangle$ and $|2\rangle$, on the other hand, it gives a kick to the atom. The coefficient of the superposition $\theta_{\text {las }}$ can be easily varied by changing the laser intensity or duration. 


\section{Detection}

We will use the following model for the detection [9]. The initial state of the atom detector is $|g\rangle$. The evolution time is divided in time steps $t_{1}, t_{2}, \ldots, t_{n}, \ldots$, of duration $\delta t \ll \Gamma^{-1}, \nu^{-1}$. After each time interval $\delta t$, the internal state of atom $C$ is measured and the state of the whole system is projected onto $|g\rangle$ or $|e\rangle$ depending on the outcome. Let us consider the case in which the detection at time $t_{1}, t_{2}, \ldots, t_{n}$ has yielded the outcome $|g\rangle$, and the detection at time $t_{n+1}$ has yielded $|e\rangle$. To lowest order in our expansion, the unnormalized state of atoms $A$ and $B$ at time $t \rightarrow \infty$ once we have made the corresponding projections will be

$$
\rho_{n}=K \lim _{t \rightarrow \infty} e^{\left(\mathcal{L}^{A}+\mathcal{L}^{B}\right)\left(t-t_{n}\right)} R\left(t_{n}\right),
$$

where $K$ is a constant that only depends on $\gamma, \tilde{\gamma}$, and $\delta t$, and

$$
\begin{aligned}
R(t)= & G\left(\mathbf{r}^{A}-\mathbf{r}\right) \sigma_{12}^{A} \rho^{A}(t) \sigma_{21}^{A} G\left(\mathbf{r}^{A}-\mathbf{r}\right)^{\dagger} \otimes \rho^{B}(t) \\
& +G\left(\mathbf{r}^{A}-\mathbf{r}\right) \sigma_{12}^{A} \rho^{A}(t) \otimes \rho^{B}(t) \sigma_{21}^{B} G\left(\mathbf{r}^{A}-\mathbf{r}\right)^{\dagger} \\
& + \text { same with } A \leftrightarrow B,
\end{aligned}
$$

with $\rho^{\alpha}(t)=e^{\mathcal{L}^{\alpha} t} \rho^{\alpha}(0)$. This expression along with other intermediate results are calculated in the Appendix.

Since we do not know a priori at which time the detection will take place, we have to perform the sum over all the operators $\rho\left(t_{n}\right)$. This sum can be transformed into an integral given the fact that $\delta t$ is smaller than any dynamical parameter corresponding to the evolution of atoms $A$ and $B$. Moreover, we also have to integrate to all positions $\mathbf{r}$ corresponding to the detector; that is, to all positions of atom $C$. By doing so, we are adding incoherently all the contributions coming from detections at different points of the detector. Finally, we have to trace over the motional states of atoms $A$ and $B$. The result, properly normalized, will give the averaged density operator provided the detector has performed a click (i.e., detected one photon).

\section{RESULTS}

\section{A. Density operator and fidelity}

As it is shown in the Appendix, the reduced density operator describing the internal state of atoms $A$ and $B$ in the case of detection can be written as the sum of two contributions

$$
\rho^{A B}=\frac{R_{1}+R_{2}}{\operatorname{tr}\left(R_{1}+R_{2}\right)},
$$

where

$$
\begin{aligned}
R_{1}= & \cos ^{2}\left(\theta_{\text {las }}\right) \sin ^{2}\left(\theta_{\text {las }}\right)\left[M^{A, A}|1,0\rangle\left\langle 1,0\left|+M^{B, B}\right| 0,1\right\rangle\langle 0,1|\right. \\
+ & \left.M^{A, B}|1,0\rangle\left\langle 0,1\left|+M^{B, A}\right| 0,1\right\rangle\langle 1,0|\right], \\
R_{2}= & \sin ^{4}\left(\theta_{\text {las }}\right)\left[\frac{\Gamma_{0}}{\Gamma} M^{A, A}|1,0\rangle\left\langle 1,0\left|+\frac{\Gamma_{0}}{\Gamma} M^{B, B}\right| 0,1\right\rangle\langle 0,1|\right. \\
& \left.+\frac{\Gamma_{1}}{\Gamma}\left(M^{A, A}+M^{B, B}\right)|1,1\rangle\langle 1,1|\right] .
\end{aligned}
$$

Here, we have defined

$$
\begin{aligned}
M^{\alpha, \beta}= & \int_{S} d \mathbf{r} \int_{0}^{\infty} d t \Gamma e^{-\Gamma t} \operatorname{tr}_{\mathrm{tp}}\left\{G\left(\mathbf{r}^{\alpha}(t)-\mathbf{r}\right) e^{i \mathbf{k}^{\alpha} \cdot \mathbf{r}^{\alpha}(0)} \rho_{\mathrm{tp}}^{A}(0)\right. \\
& \left.\times \rho_{\mathrm{tp}}^{B}(0) e^{-i \mathbf{k}^{\beta} \cdot \mathbf{r}^{\beta}(0)} G\left(\mathbf{r}^{\beta}(t)-\mathbf{r}\right)^{\dagger}\right\}
\end{aligned}
$$

where the first integral is extended to the detector surface, the trace is taken over the motional states of both atoms, and $\rho_{\mathrm{tp}}^{A, B}(0)$ denote the initial motional states (14). The timedependent operators $\mathbf{r}^{\alpha}(t)=\exp \left(i H_{\mathrm{tp}}^{\alpha} t\right) \mathbf{r}^{\alpha} \exp \left(-i H_{\mathrm{tp}}^{\alpha} t\right)$ are defined in the interaction picture with respect to the harmonic potential.

The interpretation of Eq. (18) is very simple. The term $R_{1}$ comes from processes in which only one atom is excited by the laser pulses and the subsequent photon emission is captured at the detector. This can be easily understood if one writes such a term as

$$
R_{1}=\int_{S} d \mathbf{r} \int_{0}^{\infty} d t \Gamma e^{-\Gamma t} \operatorname{tr}_{\operatorname{tp}}\{|\psi(t)\rangle\langle\psi(t)|\},
$$

with

$$
\begin{aligned}
|\psi(t)\rangle_{A, B}= & G\left(\mathbf{r}^{A}(t)-\mathbf{r}\right) e^{i \mathbf{k}^{A} \cdot \mathbf{r}^{A}(0)}|1,0\rangle_{A, B} \\
& +G\left(\mathbf{r}_{B}(t)-\mathbf{r}\right) e^{i \mathbf{k}^{B} \cdot \mathbf{r}^{B}(0)}|0,1\rangle_{A, B} .
\end{aligned}
$$

The state $|\psi(t)\rangle$ is the superposition of two states. The first one comes from the process in which at time zero the laser excites atom $A$, including the corresponding recoil; then, at time $t$ the atom emits a photon which is detected by the atomic detector at position $\mathbf{r}$. The factor $G\left[\mathbf{r}^{A}(t)-\mathbf{r}\right]$ includes the phase acquired during the propagation from the position of atom $A$ to the detector as well as the attenuation of the probability of reaching the detector which is inversely proportional to the distance traveled (a solid angle factor). The second term has the same contribution but for the process in which atom $B$ is excited. Since we do not take into account the exact time at which the photon is detected, we have to multiply $|\psi(t)\rangle\langle\psi(t)|$ by the probability density that the photon is emitted at time $t$, proportional to $e^{-\Gamma t}$, and integrate over time. On the other hand, since we do not know the point at the detector where the photon arrives, we have also to integrate the resulting expression over the detector surface, resulting in Eq. (21). Notice that retardation effects are not included in our formulation. They can be simply incorporated to this formula by changing $t \rightarrow t-\mid \mathbf{r}^{A, B}(t)$ $-\mathbf{r} / / c$. Since here $\mathbf{r}^{A, B}$ and $\mathbf{r}$ vary over very small distances (size of the atomic wave packets and detector size, respectively), the result will not be affected by retardation effects. On the other hand, expanding the term $R_{2}$ in a similar way as Eq. (21) one can readily see that it comes from the process in which both atoms are excited by laser pulses, one photon emission is detected and the other not. The terms proportional to $\Gamma_{0}$ correspond to the case in which the undetected photon is emitted in the transition $|2\rangle \rightarrow|0\rangle$, whereas the ones with $\Gamma_{1}$ correspond to the $|2\rangle \rightarrow|1\rangle$ transition.

With these expressions, we can easily calculate the fidelity (2) as 


$$
F=\frac{1}{2} \cos ^{2}\left(\theta_{\text {las }}\right)\left[1+\frac{M^{A, B} e^{i \phi}+M^{B, A} e^{-i \phi}}{M^{A, A}+M^{B, B}}\right]+\frac{\Gamma_{0}}{2 \Gamma} \sin ^{2}\left(\theta_{\text {las }}\right),
$$

where $\phi$ is the phase introduced in Eq. (1). Given the fact that the size of the atom wave packets is much smaller than $D$, we can further simplify these expressions. First, we write $\mathbf{r}^{\alpha}=\mathbf{r}_{0}^{\alpha}+\mathbf{s}^{\alpha}$ with $\left|\mathbf{r}_{\alpha}^{0}-\mathbf{r}\right| \gg \bar{s}^{\alpha}$, the typical value taken by the operator $\mathbf{s}^{\alpha}$ (of the order of the size of the atomic wave packet). Then, we expand

$$
\begin{aligned}
& G\left[\mathbf{r}^{\alpha}(t)-\mathbf{r}\right] e^{i \mathbf{k}^{\alpha} \cdot \mathbf{r}^{\alpha}(0)} \\
& \quad \simeq-\frac{e^{i\left(\mathbf{k}^{\alpha} \cdot \mathbf{r}_{0}^{\alpha}+k_{1}\left|\mathbf{r}_{0}^{\alpha}-\mathbf{r}\right|\right)}}{k_{1}\left|\mathbf{r}_{0}^{\alpha}-\mathbf{r}\right|} e^{-i \mathbf{k}_{1}^{\alpha} \cdot \mathbf{s}^{\alpha}(t)} e^{i \mathbf{k}^{\alpha} \cdot \mathbf{s}^{\alpha}(0)},
\end{aligned}
$$

where $\mathbf{k}_{1}^{\alpha}$ is a vector of modulus $k_{1}=2 \pi / \lambda_{1}$ and direction given by $\mathbf{r}-\mathbf{r}_{0}^{\alpha}$. The integrals extended to the detector in Eq. (20) can then be performed using standard methods of classical optics [substituting $\mathbf{r}$ by $\mathbf{r}_{0}$ in the denominator of Eq. (24), and expanding $\mathbf{r}$ around $\mathbf{r}_{0}$ in the exponential for $M^{A, B}$ and $\left.M^{B, A}\right]$. Taking for simplicity $\left|\mathbf{r}_{0}^{A}-\mathbf{r}_{0}\right|=\left|\mathbf{r}_{0}^{B}-\mathbf{r}_{0}\right|=\left(d^{2}\right.$ $\left.+D^{2}\right)^{1 / 2}$ we find $M^{A, A}=M^{B, B}=L_{x} L_{y} /\left(d^{2}+D^{2}\right)$ and

$$
M^{A, B}=\left(M^{B, A}\right)^{*}=M^{A, A} e^{i\left(\mathbf{k}^{A} \cdot \mathbf{r}_{0}^{A}-\mathbf{k}^{B} \cdot \mathbf{r}_{0}^{B}\right)} F_{\mathrm{geo}} F_{\mathrm{dyn}},
$$

where

$$
\begin{aligned}
F_{\mathrm{geo}}= & \frac{1}{L_{x} L_{y}} \int_{-L_{x} / 2}^{L_{x} / 2} d x \int_{-L_{y} / 2}^{L_{y} / 2} d y e^{-i k_{1} x d /\left(d^{2}+D^{2}\right)^{1 / 2}} \\
F_{\mathrm{dyn}}= & \Gamma \int_{0}^{\infty} d t e^{-\Gamma t} \operatorname{tr}_{\mathrm{tp}}\left\{e^{-i \mathbf{k}_{1}^{A} \cdot \mathbf{s}^{A}(t)} e^{-i \mathbf{k}^{A} \cdot \mathbf{s}^{A}(0)} \rho_{\mathrm{tp}}^{A}(0)\right\} \\
& \times \operatorname{tr}_{\mathrm{tp}}\left\{\rho_{\mathrm{tp}}^{B}(0) e^{i \mathbf{k}^{B} \cdot \mathbf{s}^{B}(0)} e^{-i \mathbf{k}_{1}^{B} \cdot \mathbf{s}^{B}(t)}\right\} .
\end{aligned}
$$

Evidently, $F_{\text {geo }}$ coincides with Eq. (4). On the other hand, denoting by $\chi$ the angle between $\mathbf{k}_{1}^{A}$ and $\mathbf{k}^{A}$, which for simplicity we take to be equal to the angle between $\mathbf{k}_{1}^{B}$ and $\mathbf{k}^{B}$, we obtain Eq. (5). By further choosing $\phi=-\left(\mathbf{k}_{A} \cdot \mathbf{r}_{A}^{0}\right.$ $-\mathbf{k}_{B} \cdot \mathbf{r}_{B}^{0}$ ) we obtain

$$
F=\frac{1}{2} \cos ^{2}\left(\theta_{\text {las }}\right)\left[1+F_{\text {geo }} F_{\text {dyn }}\right]+\frac{\Gamma_{0}}{2 \Gamma} \sin ^{2}\left(\theta_{\text {las }}\right) .
$$

Taking the worst case $\Gamma_{0}=0$, we finally arrive at Eq. (3).

\section{B. Detection probability}

In order to derive an expression for the detection probability we just have to combine geometrical considerations with the detection efficiency $\eta_{D}$ and the excitation probability. The probability of detection of an emitted photon is given by

$$
P_{0}=\eta_{D} \frac{D}{\left(d^{2}+D^{2}\right)^{1 / 2}} \frac{L_{x} L_{y}}{4 \pi\left(d^{2}+D^{2}\right)}
$$

$\eta_{D}$ being the quantum efficiency of the photon detector. The first quotient in the expression is the cosine of the angle between the vector connecting the atoms and the center of the detector with a vector perpendicular to its surface. The second one is the solid angle extended by the detector from the atomic positions. The probability that one and only one atom is excited and the corresponding emitted photon detected is $P_{0} 2 \sin ^{2}\left(\theta_{\text {las }}\right) \cos ^{2}\left(\theta_{\text {las }}\right)$. The probability that both atoms are excited and one of the emitted photons is detected is $P_{0} 2 \sin ^{4}\left(\theta_{\text {las }}\right)$ (we neglect the process in which both photons go to the detector). Thus, the desired probability is

$$
P_{\mathrm{det}}=\sin ^{2}\left(\theta_{\text {las }}\right) \eta_{D} \frac{D L_{x} L_{y}}{2 \pi\left(d^{2}+D^{2}\right)^{3 / 2}} .
$$

The maximum probability occurs for $D=d / \sqrt{2}$.

\section{DISCUSSION}

As shown in the previous sections, using our proposal, one can create states close to the maximally entangled state (1). A typical test to determine whether one has succeeded or not, such as searching for violations of the $\mathrm{CHSH}$ inequalities [1], would require the repetition of the experiment several times, and different measurements on the internal atomic states. A positive result would occur if $F \gtrsim 0.79$, something imposing restrictive conditions on the parameters of the experimental setup.

To create an entangled state of high fidelity the following conditions are required [see Eq. (3) and Eq. (6a)]: first, $\epsilon_{1}$ $\equiv \sin ^{2}\left(\theta_{\text {las }}\right) \ll 1, \quad$ second,$\quad \epsilon_{2} \equiv d L_{x} /\left[2 \lambda_{1}\left(d^{2}+D^{2}\right)^{1 / 2}\right] \ll 1$, third, either $\epsilon_{3} \equiv 2 \eta^{2} \operatorname{coth}\left(\hbar \nu / 2 k_{B} T\right)(\nu / \Gamma)^{2} \ll 1$ (weak confinement) or $\epsilon_{3} \equiv 2 \eta^{2} \operatorname{coth}\left(\hbar \nu / 2 k_{B} T\right) \ll 1 \quad$ (strong confinement). The first two conditions immediately imply a detection probability $P_{\text {det }} \ll 1$. In terms of these parameters we have

$$
\begin{gathered}
F \simeq 1-\frac{1}{2}\left[\epsilon_{1}+\frac{\epsilon_{2}^{2}}{6}+\epsilon_{3}\right], \\
P_{\text {det }}=\frac{4}{\pi} \eta_{D} \epsilon_{1} \epsilon_{2}^{3} \frac{L_{y}}{L_{x}} \frac{D}{L_{x}}\left(\frac{\lambda_{1}}{d}\right)^{3} .
\end{gathered}
$$

Choosing a favorable case such as $\epsilon_{1}=0.1, \epsilon_{2}=0.5, \epsilon_{3}=0.1$, still gives rise to a fidelity $F>0.8$ (i.e., Bell inequalities are still violated). Let us analyze for this case how "distant" the atoms can be for sensible values of the parameters. Rewriting $\epsilon_{2}$ as

$$
\epsilon_{2}=\frac{1}{2}\left[\frac{\lambda_{1}}{d} \sqrt{\left(\frac{d}{L_{x}}\right)^{2}+\left(\frac{D}{L_{x}}\right)^{2}}\right]^{-1}
$$


a value 0.5 impose $D / L_{x} \simeq 50$, assuming it is not possible $L_{x} \ll d \simeq D$ that would minimize $\epsilon_{2}$ while maximizing $P_{\operatorname{det}}$. Substituting in Eq. (30b)

$$
P_{\mathrm{det}}=0.8 \eta_{D} \frac{L_{y}}{L_{x}}\left(\frac{\lambda_{1}}{d}\right)^{3}
$$

Considering an experiment is performed every $10^{-4} \mathrm{sec}$ (as it is typically the case with trapped ions) a $P_{\text {det }}=10^{-4}$ would correspond to a detection per second. Then, with $L_{y} / L_{x}$ $=30$ and a $50 \%$ efficiency, a separation of 100 wavelengths is possible. Notice that the observation times cannot be increased arbitrarily for the deleterious effect caused by dark counts occurring at the detector increases consequently.

Still, we need to asses the feasibility of $\epsilon_{3}=0.1$ or equivalently of $F_{\text {dyn }}=0.9$. In doing so, we will define a new parameter, i.e.,

$$
\eta_{I} \equiv k_{1} \sqrt{\frac{\hbar}{2 m \Gamma}}
$$

so that $\eta^{2}=\eta_{I}^{2}(\Gamma / \nu)$. The new parameter (a redefinition of the Lamb-Dicke parameter with $\Gamma$ replacing $\nu$ ) allow us to study the behavior of $F_{\text {dyn }}$ with respect to $\nu / \Gamma$. Once an atom and transition are chosen, $\eta_{I}$ is fixed. Then, different values of $\nu / \Gamma$ corresponds to different designs of the trap for the chosen atom and transition. In the weak confinement limit, for fixed $\eta_{I}, \epsilon_{3} \sim \nu / \Gamma$ [just substitute Eq. (33) in Eq. (6b)], whereas in the strong limit $\epsilon_{3} \sim \Gamma / \nu$. In both extremes, then, $F_{\text {dyn }}$ approaches 1. However, for the former case Eq. (6b) is not valid for arbitrarily low values of $\nu / \Gamma$ unless $\cos (\chi)=1$ strictly. Any finite value of $\chi$ implies $F_{\text {dyn }}=0$ at $\nu / \Gamma=0$. Actually $\chi$ must be finite in order to avoid the laser light to impinge the detector, and therefore the best we can expect is a local maximum for $F_{\text {dyn }}$ close to 1 . On the other hand, the strong confinement limit can be illusory for dipole transitions (needed to detection of the spontaneously emitted photon in a reasonable time). We are bounded, then, to treat $F_{\text {dyn }}$ exactly. In Fig. 2 the behavior of the dynamical factor with respect to $\nu / \Gamma$ is displayed for two values of $\eta_{I}$, namely, 0.05 [Fig. 2(a)] and 0.3 [Fig. 2(b)]. The value $\eta_{I}$ $=0.05$ corresponds approximately to the case of the NIST experiment $[5,6]$. The $\chi$ angle has been set to $8^{\circ}$, far larger than the minimum needed to avoid the laser light to impinge on the detector $\left(0.8^{\circ}\right.$ for $\left.D / L_{x} \simeq 50\right)$. In both figures the optimum case of side band cooling reaching $T=0$ is compared with standard laser cooling at the Doppler limit and half the way to it. The maximum of $\nu / \Gamma$ is set to 1 , corresponding to the trap frequency equaling a dipole transition decay rate. The value $\eta_{I}=0.3$ represents in such a case a limit for Doppler cooling reaching $\epsilon_{3}=0.1$. From the curves shown, Doppler cooling is far enough for guaranteeing $F_{\text {dyn }}$ $\geqslant 0.9$ with sensible values of $\nu / \Gamma$.

The main problem which makes the detection probability small and prevents the creation of a macroscopic distant entangled state is the geometrical factor. The factor referring to the laser pulse area simply reduces by a factor of 10 the detection probability. In order to reduce the effects of the geometrical factor, one can use lenses to collect photons emitted in different directions. One could also couple the atoms to optical fibers, which would allow us to create en-

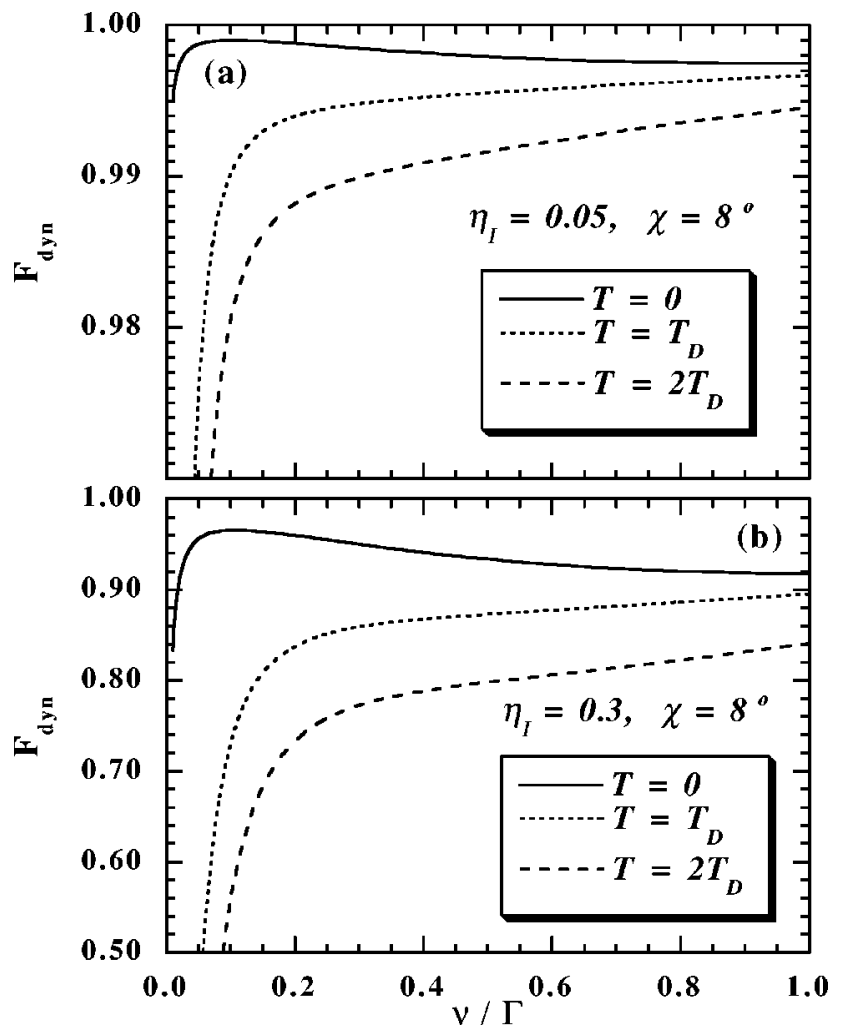

FIG. 2. The behavior of $F_{\text {dyn }}$ as a function of $\nu / \Gamma$ for two different values of $\eta_{I}$ and three different temperatures. $T_{D}$ denotes the Doppler limit temperature, i.e., $T_{D}=\hbar \Gamma / 2 k_{B}$.

tangled atoms over longer distances. In fact, one could embed the atoms in optical cavities, so that, with a high probability the emitted photons, would go to the cavity mode, and then to a fiber coupled to it. The extent to which this can be performed in practice depends on (near) future developments in cavity QED.

One can easily generalize the scheme proposed here to the case of more atoms. For example, one can take $N$ atoms, excite all of them weakly using a short laser pulse, and wait for a photodetection. In the ideal case, a state

$$
\begin{aligned}
|\psi\rangle= & \frac{1}{\sqrt{N}}\left(e^{i \phi_{1}}|1,0,0, \ldots, 0\rangle+e^{i \phi_{2}}|0,1,0, \ldots, 0\rangle\right. \\
& \left.+\cdots+e^{i \phi_{N}}|0,0,0, \ldots, 1\rangle\right)
\end{aligned}
$$

would be created. By using more photodetectors and observing more detection events one could create more general entangled states, although with a decreasing probability of success.

Note added in proof. It has been pointed out to us by W. Itano (NIST, Boulder) that the creation of entangled states of two atoms after emission of one photon was discussed by R. H. Dicke [10].

\section{ACKNOWLEDGMENTS}

C. Cabrillo acknowledges the hospitality from the University of Innsbruck. This work was supported in part by the Acciones Integradas No. HU/997-0030 (Spain-Austria), 
Grant Nos. TIC95-0563-C05-03 and PB96-00819 from CICYT (Spain), the Comunidad de Madrid under Grant No. 06T/039/96 (Spain), the FWF (Austrian Science Foundation), and TMR Network No. ERB-FMRX-CT96-0087.

\section{APPENDIX: CALCULATION OF $\rho^{A B}$}

Let us denote by $\mathcal{S}(t)$ the free evolution operator, i.e.,

$$
\mathcal{S}(t)=e^{\left(\mathcal{L}^{A}+\mathcal{L}^{B}+\mathcal{L}^{C}\right) t}
$$

Then, iterating twice, Eq. (12) results in

$$
\begin{aligned}
\rho(t)= & \mathcal{S}\left(t-t_{0}\right) \rho\left(t_{0}\right)+\int_{t_{0}}^{t} d \tau \mathcal{S}(t-\tau)\left[\left(\mathcal{S}^{A, C}+\mathcal{J}^{A, C}\right)\right. \\
& \left.\times \mathcal{S}(\tau) \rho\left(t_{0}\right)+A \leftrightarrow B\right]+\int_{t_{0}}^{t} d \tau \int_{t_{0}}^{\tau} d \tau^{\prime} \mathcal{S}(t-\tau)\left[\left(\mathcal{S}^{A, C}\right.\right. \\
& \left.+\mathcal{J}^{A, C}\right) \mathcal{S}\left(t-\tau^{\prime}\right)\left\{\left(\mathcal{S}^{A, C}+\mathcal{J}^{A, C}\right) \mathcal{S}\left(\tau^{\prime}\right) \rho\left(t_{0}\right)\right. \\
& +A \leftrightarrow B\}]+A \leftrightarrow B+O\left[\left(\mathcal{S}^{\alpha, C}\right)^{3}\right] .
\end{aligned}
$$

We are here interested only in its projection onto the detector atom excited state, i.e., in $\langle e|\rho(t)| e\rangle$. The free evolution of the detector atom is governed by

$$
\begin{gathered}
e^{\mathcal{L}^{C}{ }_{t} \sigma_{g g}^{C}=\sigma_{g g}^{C},} \\
e^{\mathcal{L}^{C}{ }^{C} \sigma_{e g}^{C}=e^{-t \gamma / 2} \sigma_{e g}^{C},} \\
e^{\mathcal{L}^{C}{ }^{C} \sigma_{g e}^{C}}=e^{-t \gamma / 2} \sigma_{g e}^{C}, \\
e^{\mathcal{L}^{C}{ }_{t}} \sigma_{e e}^{C}=e^{-t \gamma} \sigma_{e e}^{C},
\end{gathered}
$$

and it is simply enough to be operated out of $\langle e|\rho(t)| e\rangle$ given the initial state $\rho\left(t_{0}\right)=\tilde{\rho}^{A}\left(t_{0}\right) \otimes \tilde{\rho}^{B}\left(t_{0}\right) \otimes \sigma_{g g}^{C}$. Thus,

$$
\begin{aligned}
& \left\langle e\left|\left[\mathcal{S}\left(t-t_{0}\right) \rho\left(t_{0}\right)\right]\right| e\right\rangle \\
& \quad=\left\langle e\left|\mathcal{S}(t-\tau)\left[\left(\mathcal{S}^{\alpha, C}+\mathcal{J}^{\alpha, C}\right) \mathcal{S}(\tau) \rho\left(t_{0}\right)\right]\right| e\right\rangle=0
\end{aligned}
$$

so that

$$
\begin{aligned}
& \langle e|\rho(t)| e\rangle=\int_{t_{0}}^{t} d \tau \int_{t_{0}}^{\tau} d \tau^{\prime} e^{-\gamma(t-\tau)} e^{-\gamma / 2\left(\tau-\tau^{\prime}\right)} \times\langle e| e^{\left(\mathcal{L}^{A}+\mathcal{L}^{B}\right)(t-\tau)}\left[\mathcal { S } ^ { A , C } e ^ { ( \mathcal { L } ^ { A } + \mathcal { L } ^ { B } ) ( \tau - \tau ^ { \prime } ) } \left\{\mathcal{S}^{A, C} e^{\left(\mathcal{L}^{A}+\mathcal{L}^{B}\right) \tau^{\prime}} \rho\left(t_{0}\right)\right.\right. \\
& \left.\left.+\mathcal{S}^{B, C} e^{\left(\mathcal{L}^{A}+\mathcal{L}^{B}\right) \tau^{\prime}} \rho\left(t_{0}\right)\right\}\right]|e\rangle+A \leftrightarrow B
\end{aligned}
$$

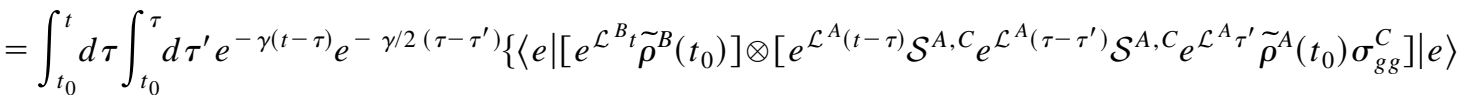

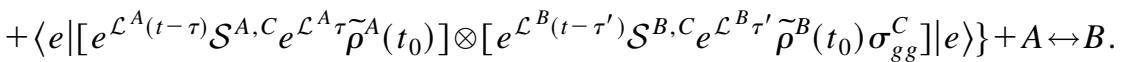

As explained in the text during the measurement process, the detector atom is projected $n$ times onto the ground state before being projected onto the excited state at time $t_{n+1}$. Under the condition $\delta t \ll \Gamma^{-1}, \nu^{-1}$ the evolution Liouvillians inside Eq. (A4) between $t_{n}$ and $t_{n+1}$ can be left constant so that

$$
\begin{aligned}
\langle e|\rho| e\rangle & \propto \int_{t_{n}}^{t_{n+1}} d \tau \int_{t_{n}}^{\tau} d \tau^{\prime} e^{-\gamma(t-\tau)} e^{-\gamma / 2\left(\tau-\tau^{\prime}\right)} \widetilde{R}\left(t_{n}\right) \\
& =\frac{2}{\gamma^{2}}\left[1+e^{-\gamma \delta t}-2 e^{-\gamma \delta t / 2}\right] \widetilde{R}\left(t_{n}\right)
\end{aligned}
$$

where

$$
\begin{aligned}
& \widetilde{R}\left(t_{n}\right)=\left\langle e\left|\left[e^{\mathcal{L}^{B} t_{n}} \widetilde{\boldsymbol{\rho}}^{B}\left(t_{0}\right)\right] \otimes\left[\mathcal{S}^{A, C} \mathcal{S}^{A, C} e^{\mathcal{L}^{A} t_{n}} \widetilde{\boldsymbol{\rho}}^{A}\left(t_{0}\right) \sigma_{g g}^{C}\right]\right| e\right\rangle \\
& +\left\langle e\left|\left[\mathcal{S}^{A, C} \mathcal{S}^{B, C} e^{\left(\mathcal{L}^{A}+\mathcal{L}^{B}\right) t_{n}} \rho\left(t_{0}\right)\right]\right| e\right\rangle+A \leftrightarrow B .
\end{aligned}
$$

Substituting the definitions of $\mathcal{S}^{\alpha, C}$ in the previous equation, changing $t_{n}$ by $t$ and denoting $e^{\mathcal{L}^{\alpha} t} \widetilde{\rho}^{\alpha}(0)$ by $\rho^{\alpha}(t)$ one arrives to an expression proportional to Eq. (17).
To proceed further we need to integrate the free evolution of the atoms given by

$$
\begin{aligned}
\dot{\rho}^{\alpha}= & -i\left[H_{t p}, \rho^{\alpha}\right]-\frac{\Gamma}{2} \sigma_{22}^{\alpha} \rho^{\alpha}-\frac{\Gamma}{2} \rho^{\alpha} \sigma_{22}^{\alpha} \\
& +\Gamma_{0} \int d \Omega e^{-i \mathbf{k}_{0}(\Omega) \cdot \mathbf{r}^{\alpha}} \sigma_{02}^{\alpha} \rho^{\alpha} \sigma_{20}^{\alpha} e^{i \mathbf{k}_{0}(\Omega) \cdot \mathbf{r}^{\alpha}}+0 \leftrightarrow 1
\end{aligned}
$$

In a frame rotating with the trap Liouvillian and assuming $t_{0} \equiv 0$ from now on the solution results in

$$
\begin{aligned}
\rho^{\alpha}(t)= & e^{-\sigma_{22}^{\alpha} t \Gamma / 2} \rho^{\alpha}(0) e^{-\sigma_{22}^{\alpha} t \Gamma / 2} \\
& +\Gamma_{0} \int_{0}^{t} d \tau e^{-\Gamma \tau} \int d \Omega e^{-i \mathbf{k}_{0}(\Omega) \cdot \mathbf{r}^{\alpha}(\tau)} \\
& \times \sigma_{02}^{\alpha} \rho^{\alpha}(0) \sigma_{20}^{\alpha} e^{i \mathbf{k}_{0}(\Omega) \cdot \mathbf{r}^{\alpha}(\tau)}+0 \leftrightarrow 1 .
\end{aligned}
$$

Taking into the account the initial condition (13) we have 


$$
\begin{aligned}
& \lim _{t \rightarrow \infty} e^{\left(\mathcal{L}^{A}+\mathcal{L}^{B}\right)(t-\tau)} R(\tau)=\left[\cos ^{2}\left(\theta_{\text {las }}\right) \sigma_{00}^{A} \otimes \rho_{t p}^{A}(0)\right. \\
& +\sin ^{2}\left(\theta_{\mathrm{las}}\right) \sigma_{00}^{A} \Gamma_{0} \int_{0}^{\infty} d \tau^{\prime} e^{-\Gamma \tau^{\prime}} \int d \Omega e^{-i \mathbf{k}_{0}(\Omega) \cdot \mathbf{r}^{B}\left(\tau^{\prime}\right)} e^{i \mathbf{k}^{B} \cdot \mathbf{r}^{B}(0)} \rho_{\mathrm{tp}}^{B}(0) e^{-i \mathbf{k}^{B} \cdot \mathbf{r}^{B}(0)} e^{i \mathbf{k}_{0}(\Omega) \cdot \mathbf{r}^{B}\left(\tau^{\prime}\right)} \\
& \left.+\sin ^{2}\left(\theta_{\text {las }}\right) \sigma_{11}^{A} \Gamma_{1} \int_{0}^{\infty} d \tau^{\prime} e^{-\Gamma \tau^{\prime}} \int d \Omega e^{-i \mathbf{k}_{1}(\Omega) \cdot \mathbf{r}^{B}\left(\tau^{\prime}\right)} e^{i \mathbf{k}^{B} \cdot \mathbf{r}^{B}(0)} \rho_{\mathrm{tp}}^{B}(0) e^{-i \mathbf{k}^{B} \cdot \mathbf{r}^{B}(0)} e^{i \mathbf{k}_{1}(\Omega) \cdot \mathbf{r}^{B}\left(\tau^{\prime}\right)}\right] \\
& \otimes \sin ^{2}\left(\theta_{\text {las }}\right) e^{-\Gamma \tau} \sigma_{11}^{A} G\left(\mathbf{r}^{A}(\tau)-\mathbf{r}\right) e^{i \mathbf{k}^{A} \cdot \mathbf{r}^{A}(0)} \rho_{\text {tp }}^{A}(0) e^{-i \mathbf{k}^{A} \cdot \mathbf{r}^{A}(0)} G\left(\mathbf{r}^{A}(\tau)-\mathbf{r}\right)^{\dagger} \\
& +\sin ^{2}\left(\theta_{\text {las }}\right) \cos ^{2}\left(\theta_{\text {las }}\right) e^{-\Gamma \tau} \sigma_{10}^{A} \otimes \sigma_{01}^{B} G\left(\mathbf{r}^{A}(\tau)-\mathbf{r}\right) \rho_{\text {tp }}^{A}(0) \rho_{\text {tp }}^{B}(0) G\left(\mathbf{r}^{B}(\tau)-\mathbf{r}\right)^{\dagger}+A \leftrightarrow B .
\end{aligned}
$$

Rearranging terms, Eq. (A8) can be decomposed as $R_{1}(\tau)+R_{2}(\tau)$ with

$$
\begin{aligned}
& R_{1}(\tau)= \sin ^{2}\left(\theta_{\mathrm{las}}\right) \cos ^{2}\left(\theta_{\mathrm{las}}\right) e^{-\Gamma \tau} \times\left[G\left(\mathbf{r}^{A}(\tau)-\mathbf{r}\right) e^{i \mathbf{k}^{A} \cdot \mathbf{r}^{A}(0)}|1,0\rangle+G\left(\mathbf{r}^{B}(\tau)-\mathbf{r}\right) e^{-i \mathbf{k}_{B} \cdot \mathbf{r}^{B}(0)}|0,1\rangle\right] \rho_{\mathrm{tp}}^{A} \rho_{\mathrm{tp}}^{B} \\
& \times\left[G\left(\mathbf{r}^{A}(\tau)-\mathbf{r}\right) e^{i \mathbf{k}^{A} \cdot \mathbf{r}^{A}(0)}|1,0\rangle+G\left(\mathbf{r}^{B}(\tau)-\mathbf{r}\right) e^{-i \mathbf{k}^{B} \mathbf{r}^{B}(0)}|0,1\rangle\right]^{\dagger} \\
& R_{2}(\tau)= \sin ^{4}\left(\theta_{\mathrm{las}}\right) e^{-\Gamma \tau} \times\left\{\sigma_{11}^{A} \otimes \sigma_{00}^{B} \Gamma_{0} \int_{0}^{\infty} d \tau^{\prime} e^{-\Gamma \tau^{\prime}} \int d \Omega e^{-i \mathbf{k}_{0}(\Omega) \cdot \mathbf{r}^{B}\left(\tau^{\prime}\right)} e^{i \mathbf{k}^{B} \cdot \mathbf{r}^{B}(0)} \rho_{\mathrm{tp}}^{B}(0) e^{-i \mathbf{k}^{B} \cdot \mathbf{r}^{B}(0)} e^{i \mathbf{k}_{0}(\Omega) \cdot \mathbf{r}^{B}\left(\tau^{\prime}\right)}\right. \\
& \otimes \\
& G\left(\mathbf{r}^{A}(\tau)-\mathbf{r}\right) e^{i \mathbf{k}^{A} \cdot \mathbf{r}^{A}(0)} \rho_{\mathrm{tp}}^{A}(0) e^{-i \mathbf{k}^{A} \cdot \mathbf{r}^{A}(0)} G\left(\mathbf{r}^{A}(\tau)-\mathbf{r}\right)^{\dagger} \sigma_{11}^{A} \\
& \otimes \sigma_{11}^{B} \Gamma_{1} \int_{0}^{\infty} d \tau^{\prime} e^{-\Gamma \tau^{\prime}} \int d \Omega e^{-i \mathbf{k}_{1}(\Omega) \cdot \mathbf{r}^{B}\left(\tau^{\prime}\right)} e^{i \mathbf{k}^{B} \cdot \mathbf{r}^{B}(0)} \rho_{\mathrm{tp}}^{B}(0) e^{-i \mathbf{k}^{B} \cdot \mathbf{r}^{B}(0)} e^{i \mathbf{k}_{1}(\Omega) \cdot \mathbf{r}^{B}\left(\tau^{\prime}\right)} \\
& \otimes\left.G\left(\mathbf{r}^{A}(\tau)-\mathbf{r}\right) e^{i \mathbf{k}^{A} \cdot \mathbf{r}^{A}(0)} \rho_{\mathrm{tp}}^{A}(0) e^{-i \mathbf{k}^{A} \cdot \mathbf{r}^{A}(0)} G\left(\mathbf{r}^{A}(\tau)-\mathbf{r}\right)^{\dagger}+A \leftrightarrow B\right\}
\end{aligned}
$$

Tracing over the motional states and using the cyclic property of the trace the exponential terms in $R_{2}(\tau)$ cancel out making the integral in $\tau^{\prime}$ trivial. Integrating $\mathbf{r}$ over the detector area and $\tau$ with a density $\Gamma e^{-\Gamma \tau}, R_{1}$, and $R_{2}$ are finally obtained.

[1] J. S. Bell, Physics (Long Island City, NY) 1, 195 (1965); J. F. Clauser, M. A. Horne, A. Symony, and R. A. Holt, Phys. Rev. Lett. 23, 880 (1969); J. F. Clauser and M. A. Horne, Phys. Rev. D 10, 526 (1974).

[2] See, for example, D. P. DiVincenzo, Science 270, 255 (1995); C. H. Bennett, Phys. Today 48 (10), 24 (1995).

[3] J. I. Cirac and P. Zoller, Phys. Rev. A 50, R2799 (1994); E. Hagley, X. Mantre, G. Nogues, C. Wunderlich, M. Brune, J. M. Raimond, and S. Haroche, Phys. Rev. Lett. 79, 1 (1997); X. Mantre, E. Hagley, G. Nogues, C. Wunderlich, P. Goy, M. Brune, J. M. Raimond, and S. Haroche ibid. 79, 769 (1997); T. Pellizzari, S. Gardiner, J. I. Cirac, and P. Zoller, ibid. 75, 3788 (1995); Q. A. Turchette, C. J. Hood, W. Lange, H. Mabuchi, and H. J. Kimble, ibid. 75, 4710 (1995).

[4] J. I. Cirac and P. Zoller, Phys. Rev. Lett. 74, 4091 (1995); C. Monroe, D. M. Meekhof, B. E. King, W. M. Itano, and D. J. Wineland, ibid. 75, 4714 (1995); B. E. King, C. S. Wood, C. J. Myatt, Q. A. Turchette, D. Leibfried, W. M. Itano, C. Monroe, and D. J. Wineland, ibid. 81, 1525 (1998); J. F. Poyatos, J. I. Cirac, and P. Zoller, ibid. 81, 1322 (1998).

[5] U. Eichmann, J. C. Bergquist, J. J. Bollinger, J. M. Gilligan, W. M. Itano, D. J. Wineland, and M. G. Raizen, Phys. Rev. Lett. 70, 2359 (1993).
[6] For a detailed theoretical description of the experiment, see W. M. Itano, J. C. Bergquist, J. J. Bollinger, D. J. Wineland, U. Eichmann, and M. G. Raizen, Phys. Rev. A 57, 4176 (1998); see also, M. O. Scully and K. Drül, ibid. 25, 2208 (1982); H. Huang, G. S. Agarwal, and M. O. Scully, Opt. Commun. 127, 243 (1996); T. Wong, S. M. Tan, M. J. Collet, and D. F. Walls, Phys. Rev. A 55, 1288 (1997); R. G. Brewer, ibid. 52, 2965 (1995); 53, 2903 (1996); Phys. Rev. Lett. 77, 5153 (1996).

[7] M. Brune, E. Hagley, J. Dreyer, X. Mantre, A. Maali, C. Wunderlich, J. M. Raimond, and S. Haroche, Phys. Rev. Lett. 77, 4887 (1996).

[8] See, for example, C. Gardiner, Quantum Noise (SpringerVerlag, Berlin, 1991).

[9] C. Cohen-Tannoudji, J. Dupont-Roc, and G. Grynberg, AtomPhoton Interactions: Basic Processes and Applications (Wiley, New York, 1992).

[10] R. H. Dicke (unpublished); see also W. M. Itano, U. Eichmann, J. C. Bergquist, J. J. Bollinger, J. M. Gilligan, M. G. Raizen, and D. J. Wineland, in Proceedings of the International Conference on Lasers '93, Lake Tahoe, 1993, edited by V. J. Corcoran and T. A. Goldman (STS Press, McLean, VA, 1994), pp. 412-419. 